\title{
Rectal Probe Electrostimulation in the Treatment of Anejaculatory Spinal Cord Injured Men *
}

\author{
L. S. Halstead, M.D., ${ }^{1}$ S. VerVoort, M.D. ${ }^{2}$, S. W. J. Seager, M.A., \\ M.V.B., M.R.C.V.S. ${ }^{3}$ \\ ${ }^{1}$ Associate Professor, Departments of Rehabilitation and Physical Medicine, Baylor \\ College of Medicine, Attending Physician, The Institute for Rehabilitation and \\ Research, ${ }^{2}$ Assistant Instructor, Departments of Rehabilitation and Physical \\ Medicine, Baylor College of Medicine, ${ }^{3}$ Professor, Department of Veterinary \\ Physiology and Pharmacology, College of Veterinary Medicine, Texas AËM \\ University, U.S.A.
}

\section{Summary}

This paper reviews our experience with rectal probe electroejaculation (RPE) which is part of a larger effort to determine the correlates of successful ejaculation and fertility in SCI men. RPE is performed in the outpatient clinic using specially designed rectal probes. Over the past 18 months, we have attempted RPE on 38 occasions in 12 subjects (eight paraplegics and four quadriplegics) with an age range of 23-38 years and 0.5-18 years since onset of injury. Anterograde ejaculation occurred in nine subjects with improvement in percent motility and total live sperm count on repeated stimulations in five subjects. Significant retrograde ejaculation occurred in one person and sperm acceptable for artificial insemination $(A I)$ was obtained from four subjects. The major side effects were mild dysreflexia (three subjects) and disruption of a normal bowel program (one subject). We conclude that $R P E$ is a safe, relatively brief outpatient procedure and, with repeated stimulations, has a good potential for producing sperm acceptable for $A I$ in selected patients. Key words: Spinal cord injury (SCI); Fertility; Sexuality; Disability.

\section{Introduction}

Infertility in spinal cord injured (SCI) men is a common problem with less than $5 \%$ able to father children. The two major underlying causes of this problem are impaired ejaculation and poor sperm production. Over the years, three principal techniques have been used to induce ejaculation in SCI men: intrathecal chemical ejaculation (CE) which requires injection of chemicals into the spinal sac, electroejaculation (EE) which electrically stimulates nerves causing ejaculation, and vibratory stimulation of the penis (VS) which initiates

Correspondence to: S. M. VerVoort, M.D., 1333 Moursund Avenue, Houston, Texas 77030, U.S.A.

* Presented at the Annual Meeting of the American Spinal Injury Association, San Francisco, California, U.S.A. March 13, 1986 
reflex spinal cord activity causing ejaculation. Because of the complications associated with $\mathrm{CE}$ and the greater success of $\mathrm{EE}$ and VS, most investigators have abandoned CE [Brindley, 1984; Francois et al., 1978-79]. Although isolated reports of success with EE and VS exist, the technology and knowledge have not been sufficiently developed to permit replication in other centers.

In contrast to impaired ejaculation, the problem of poor sperm production is considerably more complex. Although likely to be multi-causal, several factors have been identified to be particularly important. These include elevated testicular temperatures, urological complications, and impaired ejaculation [Brindley 1982; Bors, Engle, et al., 1950]. At present, there is no consensus as to which etiology is the most important in the majority of cases.

Because of the complexity of these problems we have developed a multidisciplinary team with expertise in paraplegia, primate electro-ejaculation and male infertility. The general mission of this team is to evaluate in an integrated, systematic fashion, ejaculatory dysfunction and inpaired spermatogenesis. More specific goals are to: 1 ) develop a reliable technique to produce ejaculation using RPE and VS either singly or in combination; 2) undertake an in depth evaluation of the factors responsible for impaired spermatogenesis and identify possible treatments; 3) perform artificial insemination ( $\mathrm{AI}$ ) and/or sperm freezing and storage; and 4) train other physicians in these techniques so they may provide similar services in their communities. The purpose of this paper is to report our preliminary experience with rectal probe electrostimulation (RPE) in a group of 12 SCI men.

\section{Patients and methods}

This study was conducted in the Sexuality and Disability Clinic at TIRR over the past 18 months. The purpose of the clinic is to provide information and counselling, as well as support research in areas dealing with sexuality which are of concern to handicapped men and women.

Patients eligible for RPE were any men with a history of traumatic SCI who were 18 years of age or older, medically stable, and interested in determining their fertility status. Married subjects interested in pursuing AI at the time of the study were given preference, although others were included as time and resources permitted.

After providing informed consent, subjects had a complete medical history and physical examination with special attention to their neurological and urological status. Laboratory evaluations included a baseline urine culture and sensitivity, complete blood count, and total protein and albumin estimations. Subjects with urinary tract infections were treated with appropriate antibiotics and repeat urinalyses were performed on all subjects prior to each attempt at RPE.

Rectal probe electrostimulation was performed in the outpatient clinic using a $1 \frac{1}{8}$ or $1 \frac{1}{4}$-inch diameter rectal probe precision machined from solid bars of PVC. Three attached linear electrodes lie parallel to the long axis of the probe. The electrodes face anteriorly in proximity to prostatic tissue when the probe is placed in the rectum. Rectal temperature was monitored with a special sensor built into the probe and electrical stimulation was provided by a custom-made 
electric stimulator designed with isolation transformers and current-limiting devices to prevent accidental delivery of excessive current. Stimulation was begun with an on-off cycle and was limited to a maximum of 25 stimulations (60 cycles/second sine wave) of 8-15 volts and $200-400 \mathrm{~mA}$.

Prior to each ejaculation session, the subject's bladder was emptied using a sterile catheterisation technique and the rectal mucosa was examined with an illuminated rectoscope. Ejaculation procedures were usually performed with the subject in the right lateral decubitus position with a minimum of three other people present. One person performed the RPE, one monitored blood pressure and pulse at frequent intervals and recorded subject responses to stimulation, and one performed urethral massage and collected semen in a plastic cup if antegrade ejaculation occurred. Stimulations were discontinued if the blood pressure exceeded $200 \mathrm{~mm} \mathrm{Hg}$ systolic or $130 \mathrm{~mm} \mathrm{Hg}$ diastolic, or if the subject requested discontinuation because of side effects or any other reason.

At the end of each ejaculation session, an in-and-out sterile bladder irrigation was performed with normal saline to assess the presence of post-stimulation sperm in the bladder or urethra, and a repeat examination of the rectal mucosa was performed. Altogether, each session of RPE took approximately 45 minutes which included subject preparation, catheterisations, etc. The actual stimulation and semen collection time was fairly short-usually no longer than 3 to 5 minutes.

\section{Results}

We performed RPE on 38 occasions in 12 men with traumatic SCI during an 18 month period. All subjects in which electroejaculation was attempted in our clinic are included here. Four subjects had quadriplegia, eight had paraplegia, and seven were married. They ranged in age from 23 to 38 years and their duration of injury ranged from 0.5 to 18 years. Only one subject had a sphincterotomy or other urological surgery that might alter their ejaculatory mechanism. Subject number 9 had a transurethral sphincterotomy and a bladder neck resection prior to ejaculatory attempts. Urinary drainage techniques used by the subjects included reflex voiding with an external collecting device (subjects 2,3 , $5,7,8$, and 9) or intermittent catheterisation (subjects 1, 4, 6, 10,11, and 12). No subject utilised continuous urinary drainage techniques. Table I summarises the level of injury, Frankel classification, presence of deep tendon reflexes in the lower extremities, and number of attempts of RPE. The highest level of injury was C5 (subjects 1, 2, and 3) and the lowest, L1 (subject 12). Two subjects were areflexic in their legs (subjects 11 and 12). Three men had only one attempt at RPE (subjects 9, 10, and 12) while three had six or more attempts (subjects 7,8 , and 11 ).

Table II summarises the results of our RPE experience in all 12 subjects. Ten of the $12(83 \%)$ produced sperm on at least one occasion, and sperm was obtained on 32 of the 38 RPE attempts. In nine of the $12(75 \%)$ we obtained sperm in antegrade emissions, while in one person (subject 12) there was no antegrade emission, but during his single session of RPE, we found a few sperm on post-stimulation bladder washout. Of the nine subjects who had sperm on antegrade ejaculation, RPE was unsuccessful on the first attempt in three. In 
Table I Selected Characteristics of Subjects and Number of Attempts at RPE

\begin{tabular}{ccccccc}
\hline Subject & Age & $\begin{array}{c}\text { Level } \\
\text { of } \\
\text { lesion }\end{array}$ & $\begin{array}{c}\text { Frankel } \\
\text { class }\end{array}$ & $\begin{array}{c}\text { Lower } \\
\text { extremity } \\
\text { reflexes }\end{array}$ & $\begin{array}{c}\text { Duration } \\
\text { of lesion } \\
\text { (years) }\end{array}$ & $\begin{array}{c}\text { Number of } \\
\text { attempts } \\
\text { at RPE }\end{array}$ \\
\hline 1 & 25 & C5 & C & + & $1 \cdot 5$ & 4 \\
2 & 25 & C5 & C & + & 5 & 3 \\
3 & 30 & C5 & D & + & 11 & 3 \\
4 & 26 & C6 & C & + & 9 mos. & 2 \\
5 & 33 & T3 & A & + & 5 & 2 \\
6 & 27 & T4 & A & + & $11 \cdot 5$ & 3 \\
7 & 33 & T7 & A & + & 18 & 6 \\
8 & 38 & T8 & A & + & $11 \cdot 5$ & 6 \\
9 & 30 & T9 & A & + & 5 & 1 \\
10 & 32 & T10 & A & + & 3.5 & 7 \\
11 & 23 & T12 & A & - & 1 & 1 \\
12 & 28 & L1 & B & - & & 1 \\
\hline
\end{tabular}

these subjects, repeat RPE appeared to be successful in producing antegrade emission with sperm because they were more relaxed, higher voltages were used, and the investigators had a better understanding of how to achieve the threshold of stimulation necessary for ejaculation. No voiding occurred during stimulation in any of the 38 attempts of RPE. Discomfort from the electrical stimulation was experienced only by those subjects with incomplete lesions and some preservation of sensation (subjects $1,2,3,4$, and 12). No subject requested a discontinuation of the procedure because of pain.

The antegrade semen obtained demonstrated wide variations in sperm quality and quantity between subjects and in the same subject with different attempts. Three subjects (subjects 5, 6 and 10) had only rare sperm in the semen specimens, of which only one (subject 6 ) produced motile sperm. Six subjects produced semen with sperm concentrations ranging from $7 \cdot 5-678 \times 10^{6} / \mathrm{cc}$. Sperm motility ranged from $0-60^{\circ}{ }_{0}$ with $3-5+$ forward progression, and the per cent of morphologically normal sperm ranged from $0-55^{\circ}{ }_{0}$. Semen specimens ranged in volume from a few drops to $2.9 \mathrm{cc}$. Seminal fluid $\mathrm{pH}$ ranged from $6 \cdot 2-8.3$ with the majority of specimens in a neutral range $(6 \cdot 8-7 \cdot 4)$.

Table III lists the best results obtained in the nine subjects with sperm in their antegrade ejaculate. Four of these men (subjects 1, 7, 8 and 11) produced semen with live sperm counts (total sperm $\times$ per cent motility) that were adequate for artificial insemination and the spouse of one subject was inseminated on one occasion. Pregnancy was not achieved. One subject (subject 3) produced a low total sperm count $\left(6 \times 10^{6}\right)$ with $2^{\circ}{ }_{0}$ motility and $20^{\circ}{ }_{0}$ normal morphology, while another subject has a very high count $\left(950 \times 10^{6}\right)$ but no motility and only $7^{\circ}$ o normal morphology. The remaining three subjects had only rare sperm in their ejaculates which made motility and morphological evaluations impractical.

\section{Discussion}

Electroejaculation in SCI man has been attempted in various parts of the world for over 40 years. During that time, there has been a mixture of hope and 


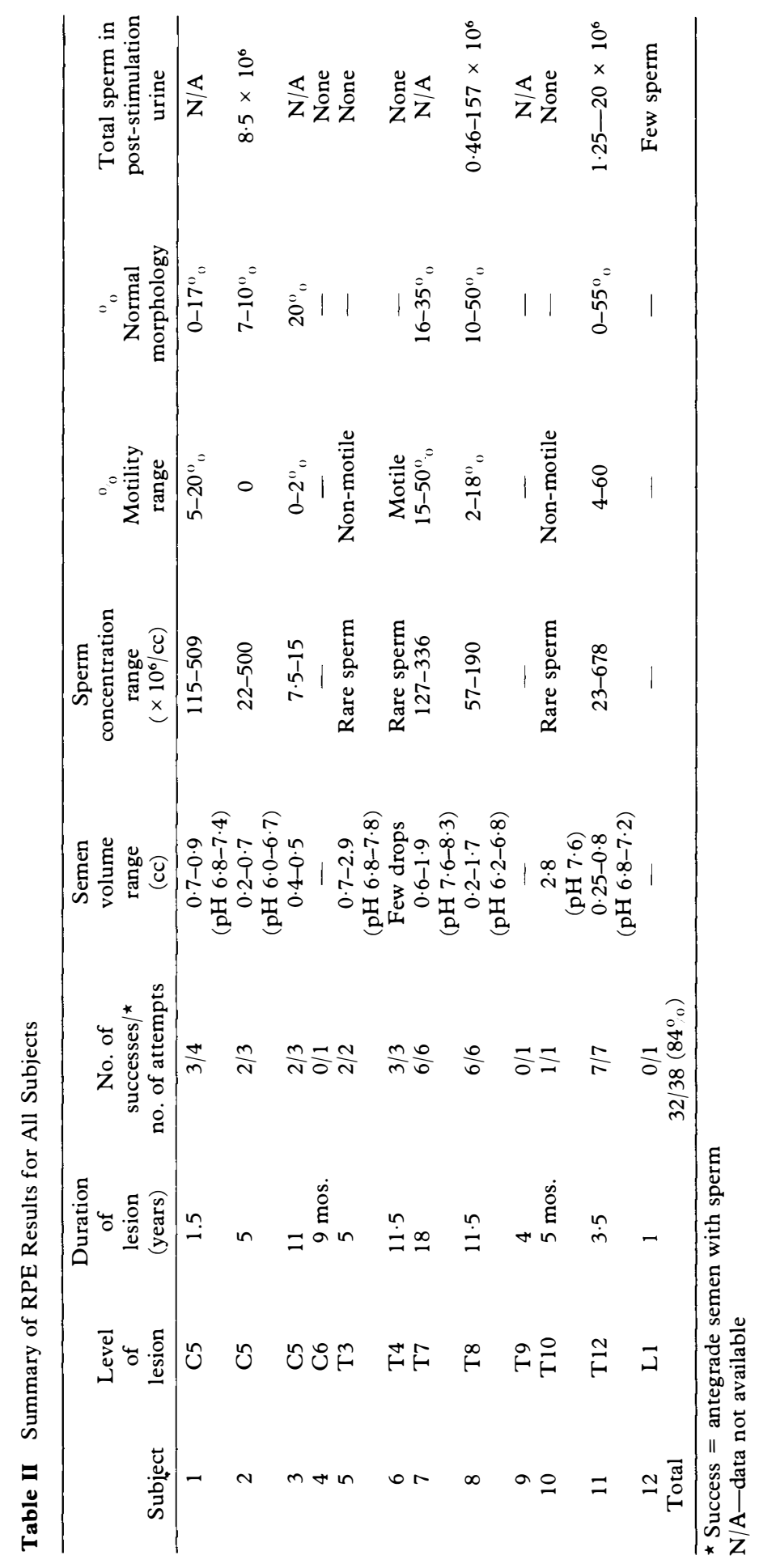




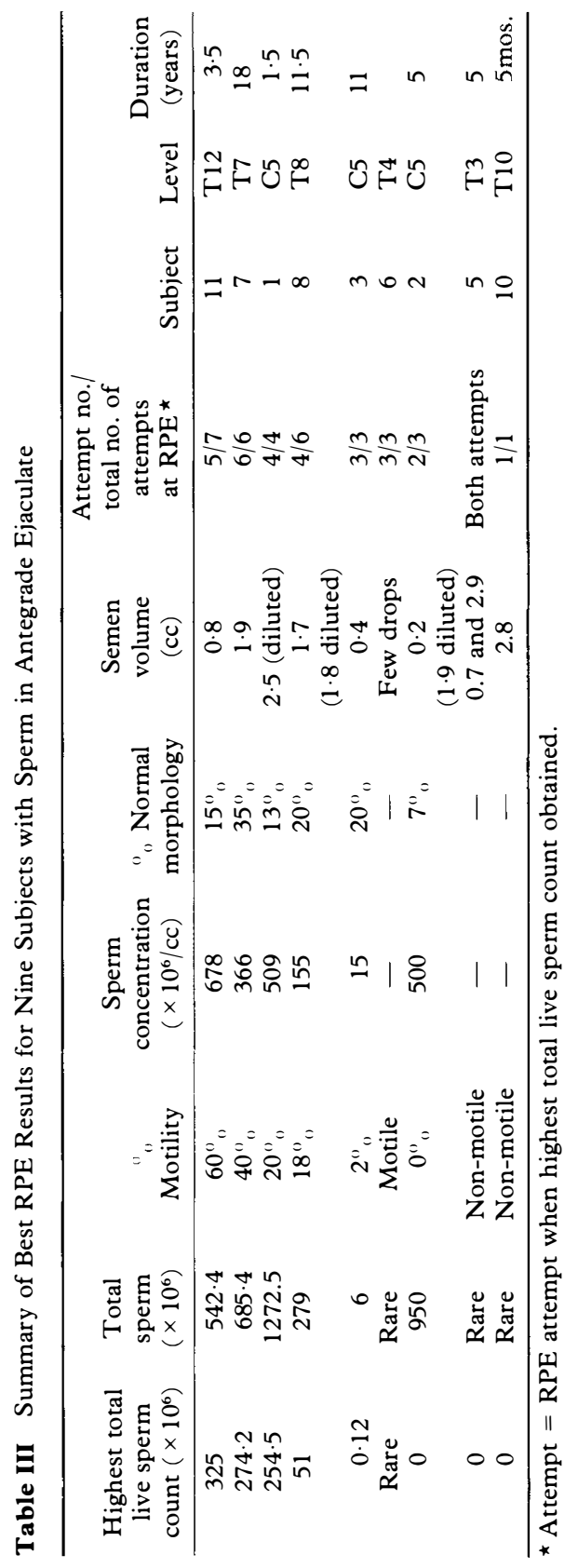




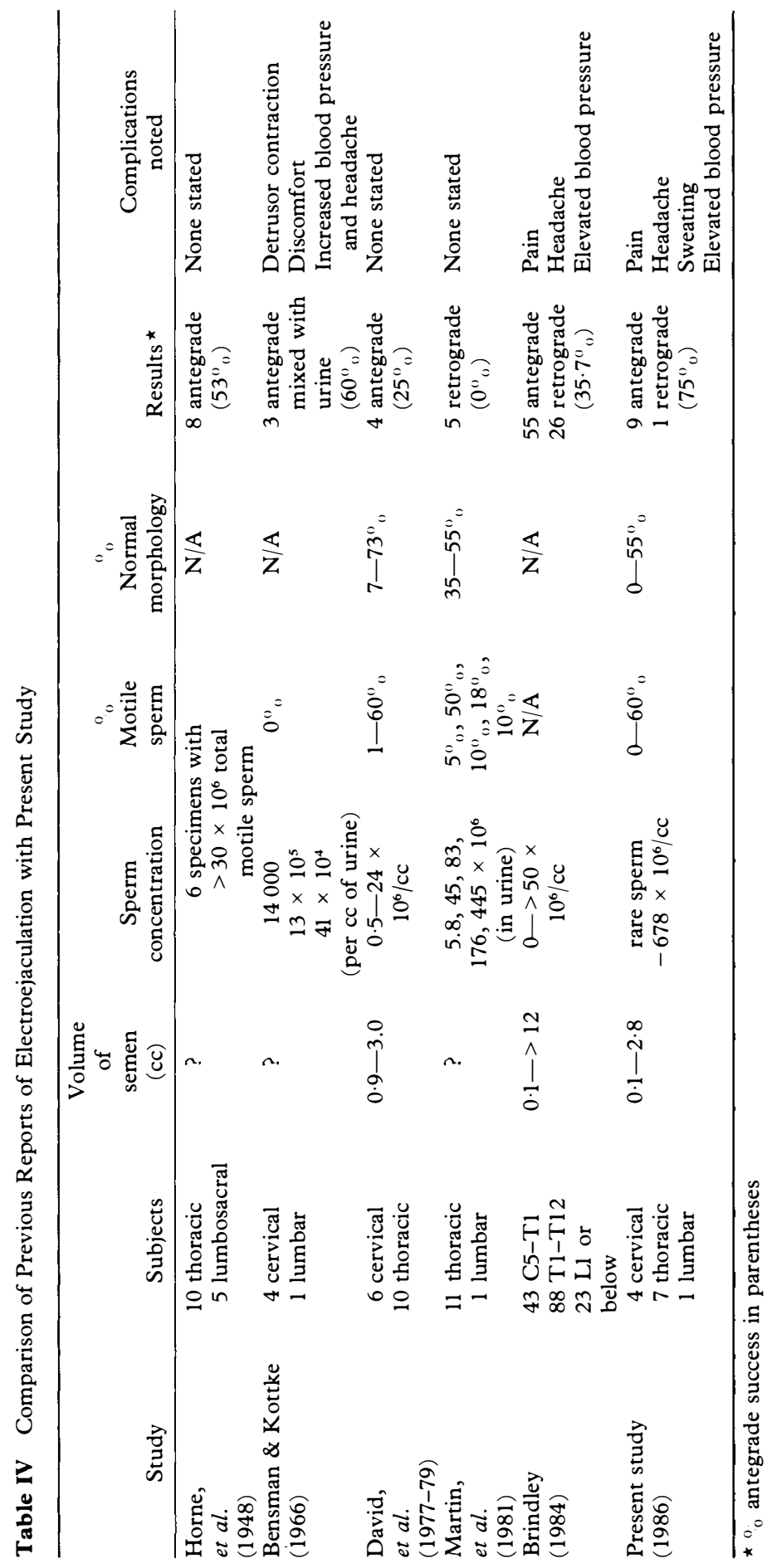


despair that a procedure which seemed relatively simple might fulfill its original promise. Despite the widespread interest and potential importance of this technique, however, it is of note that there are only five major studies on electroejaculation in the medical literature. Table IV summarises the pertinent data from these studies along with similar information from the present report.

Unfortunately, one of the difficulties in analysing the results of these other investigators is that each used different equipment and techniques along with different criteria for success. In addition, the description of the results was not always complete, so in many instances, critical pieces of data are not available for comparison. Nonetheless, some general patterns are present and it is clear that over the years encouraging progress has been made in assaulting the problem of male infertility in SCI man. While there are many problems still to be resolved, we feel that the present investigation makes an important contribution to this overall progress.

Although the number of subjects we studied was relatively small, the success rates were remarkably high and higher than in any other report. Taking the strictest definition of success, nine of 12 or $75^{\circ}{ }_{0}$, had sperm in at least one antegrade ejaculation while 10 of 12 , or $83^{\circ}$, had sperm on either antegrade emission or bladder washout. The reason for the high rate of antegrade success compared to other researchers is not clear. One explanation may be that only one of our subjects had urological surgery; another may be the type of probes and technique employed. In any event, we will need to study many more subjects before any conclusions can be drawn.

Not all subjects who were successfully ejaculated had sperm of suitable quality or quantity for artificial insemination. However, the number of subjects with sperm suitable for AI is somewhat misleading because many of our subjects participated early in the study when we were still refining our technique. For example, three of our antegrade failures (subjects 4, 9 and 12) had only one attempt at RPE and it has been our experience that the first attempt is often unsuccessful because the major focus of that session is to establish rapport with the subject and to help him become comfortable with the technique. As a result, the first RPE attempt is confined to relatively low voltages (5-10) that are not likely to produce discomfort or unpleasant side effects-or achieve the ejaculatory threshold.

The quality and quantity of sperm was highly variable both within and between subjects, as has been reported by other investigators. In addition, as with other studies, we did not find any correlation of sperm quality and quantity with level of injury, completeness, or duration of spinal cord lesion. On the contrary, some of the best specimens were obtained in subjects that initially appeared unpromising. For example, two men (subjects 7 and 8) with good specimens had been injured 18 and 11.5 years previously, and a third (subject 11) had a low thoracic injury with areflexia. Altogether, four of the 12 subjects had sperm suitable for insemination and in each instance, it was possible to obtain it on repeated RPE attempts. Thus, while we may not be any closer to understanding the causes for impaired spermatogenesis, it is encouraging to find in this sample of subjects that at least one-third had repeatable ejaculates suitable for AI.

Another finding of special interest was that the sperm counts improved in five subjects following repeated stimulations over a period of several weeks. 

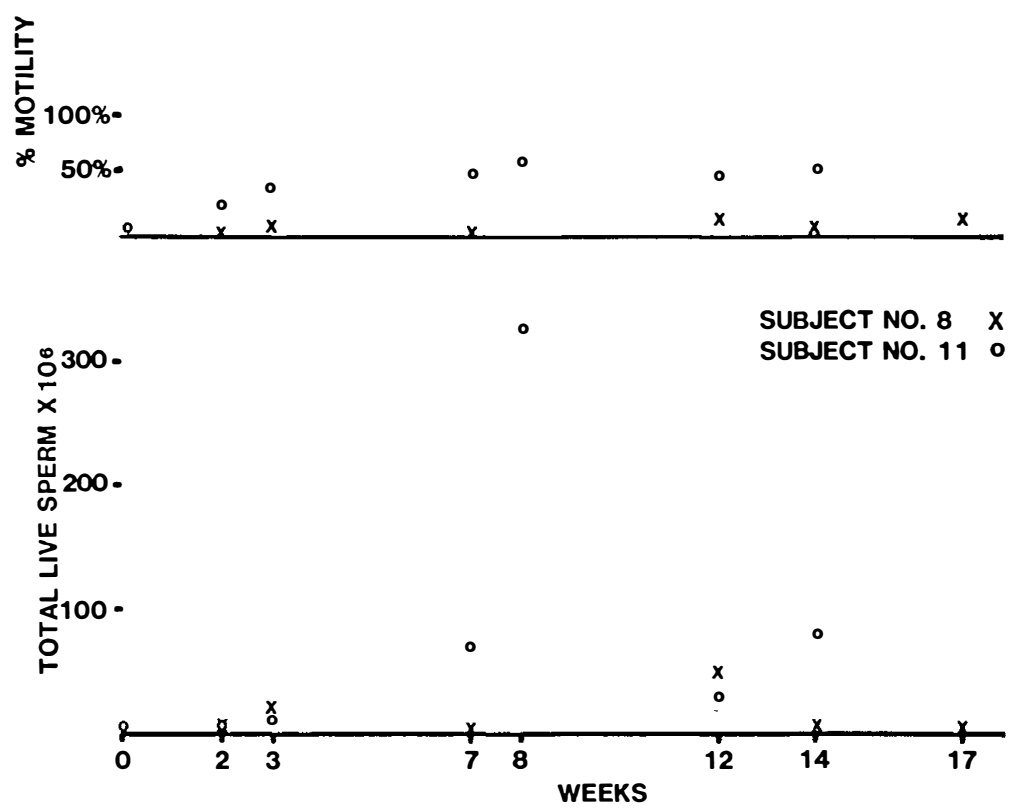

Figure 1 Per cent motility and total live sperm for a T8 and T12 paraplegic

These improvements were seen in both per cent sperm motility and per cent normal sperm morphology. Examples of these are shown in Figure 1 (subjects 8 and 11). To our knowledge, no other investigators have described evidence of improved sperm quality and quantity with repeated stimulation although some have suggested it (Brindley, 1984, Francois et al., 1978-79). In our series, all subjects who had repeated ejaculatory success demonstrated some improvement in sperm quality and quantity except subject 6 , who was ejaculated early in our study when our technique was less refined, and subject 5 , who has significant atrophy of both testicles and a history of multiple recurrent urinary tract infections.

Based on our experience, we believe there are a number of variables that contribute to successful electroejaculation, including proper equipment, a standardised technique and a relaxed and comfortable subject. However, probably the single most important factor is to have someone with extensive electroejaculatory experience. In the present study, this experience was obtained by one of us (SJS) during many years of working with neurologically intact non-human primates and, more recently, with a group of SCI monkeys. It was also apparent, as we gained more experience with human subjects, that our success rate improved and repeatable ejaculations became more predictable, which augurs well for those subjects interested in pursuing AI. Nevertheless, even in experienced hands, RPE is still associated with some side effects. In our subjects, the most common were local pain in men with incomplete lesions, and headache, sweating and elevated blood pressure in subjects prone to autonomic dysreflexia. These side effects were temporary, however, and subsided within a few minutes of discontinuing stimulation. Men with complete lesions below T6 experienced 
no adverse effects except for muscle spasms, especially in the abdomen. In addition, pre- and post-stimulation examination of the rectum revealed no mucosal damage in any subject which is the first time this finding has been reported. Because of this paucity of side effects and the relative speed with which RPE can be accomplished in the outpatient setting, subjects are able to resume their normal activities within 60 to 90 minutes of coming to the clinic.

In this first group of subjects, we have attempted one AI without success. And while the ultimate goal of our efforts is to help SCI men father children, it is not solely to achieve an occasional instance of successful AI. Rather, the real goal is to understand more clearly the causes of and treatment for impaired ejaculation and poor sperm production, and ultimately make natural fatherhood an option for all SCI men who desire it.

\section{Conclusion}

We conclude that the equipment and technique to perform successful and repeatable electroejaculation in many SCI men is now available. The next challenge is to gain a better understanding of the causes and treatment of impaired spermatogenesis. Finally, we would like to establish training programmes to instruct physicians in RPE so they may provide similar services in their communities.

\section{Acknowledgements}

This research was funded in part by a grant from the Bob and Vivian Smith Foundation, Houston, Texas, and the Model Rehabilitation Spinal Cord Injury System Grant, TIRR, funded by the National Institute of Handicapped Research.

\section{References}

Bensman A, KotTke, FJ 1966 Induced emission of sperm utilising electrical stimulation of the seminal vesicles and vas deferens. Archives of Physical Medicine and Rehabilitation 47:436-443.

Bors E, ENGLE, ET, RosENQUIST RC, et al., 1950 Fertility in paraplegic males: A preliminary report of endocrine studies. Fournal of Clinical Endocrinology and Metabolism 10:381-398.

BRINDLEY GS 1981 Reflux ejaculation under vibratory stimulation in paraplegic men. Paraplegia 19:299-302.

BRINDley GS 1984 The fertility of men with spinal injuries. Paraplegia 22:337-340.

DAvid A, OHRY A, Rozin R 1977-79 Spinal cord injuries: male infertility aspects. Paraplegia 15:11-14.

Francois N, Maury M, Jouannet D, David G, et al. 1978-79 Electroejaculation of a complete paraplegic followed by pregnancy. Paraplegia 16:248.

Horne HW, PAull DP, Monro D 1948 Fertility studies in the human male with traumatic injuries of the spinal cord and cauda equina. New England fournal of Medicine 239:959.

MARTIN DE, WARNER H, CRENSHAW TL, et al., 1983 Initiation of erection and semen release by rectal probe electrostimulation. Fournal of Urology 129:637-641. 